\title{
Early Lumen-Apposing Metal Stent Dysfunction Complicating Endoscopic Ultrasound-Guided Gastroenterostomy: A Report of Two Cases
}

\author{
Janine B. Kastelijn ${ }^{1}$, Veronique Van der Voort ${ }^{2}$, Alderina Bijlsma ${ }^{3}$, Leon M. G. Moons ${ }^{1}$, Matthijs P. Schwartz ${ }^{2}$ and Frank P. Vleggaar ${ }^{1}$ \\ Department of Gastroenterology and Hepatology, ${ }^{1}$ University Medical Center Utrecht, Utrecht, ${ }^{2}$ Meander Medical Center, Amersfoort, \\ ${ }^{3}$ Martini Hospital, Groningen, The Netherlands
}

Endoscopic ultrasonography-guided gastroenterostomy using a lumen-apposing metal stent has emerged as a novel technique in the palliative treatment of malignant gastric outlet obstruction. Endoscopic ultrasonography-guided gastroenterostomy seems to have the potential to provide long-lasting patency in a minimally invasive manner. Low reintervention rates have been described. We report two cases with early lumen-apposing metal stent dysfunction, compromising patency. One case showed food impaction after three weeks, and hyperplastic tissue overgrowth with a buried distal flange six weeks after stent placement. The latter was successfully treated by argon plasma coagulation, stent removal, and deployment of a larger-diameter lumen-apposing metal stent. The second case showed a narrowed luminal diameter of the stent and jejunal pressure ulcerations after three weeks. The narrowing was successfully treated by balloon dilation. Eight weeks later, hyperplastic tissue overgrowth at the distal flange of the stent and a gastro-colonic fistula were diagnosed, followed by extensive reconstructive surgery. Clin Endosc 2021;54:603-607

Key Words: Endoscopy; Gastric outlet obstruction; Gastroenterostomy; Palliative care; Stents

\section{INTRODUCTION}

Malignant gastric outlet obstruction (GOO) is a common complication in patients with advanced primary or metastatic malignancies located in the distal gastric or peripancreatic region. Standard treatment options for malignant GOO are duodenal stenting or surgical gastrojejunostomy. Both are effective treatments, but also have their disadvantages: duodenal stenting is minimally invasive, but has a significant risk of re-obstruction requiring reinterventions, while surgical

Received: July 28, 2020 Revised: September 21, 2020

Accepted: October 15, 2020

Correspondence: Frank P. Vleggaar

Department of Gastroenterology and Hepatology, University Medical Center Utrecht, 3508 GA, Utrecht 85500, The Netherlands

Tel: +31-88-755-1309, Fax: +31-88-755-5081, E-mail: f.vleggaar@umcutrecht.nl ORCID: https://orcid.org/0000-0001-8664-5130

(c) This is an Open Access article distributed under the terms of the Creative Commons Attribution Non-Commercial License (http://creativecommons.org/ licenses/by-nc/3.0) which permits unrestricted non-commercial use, distribution, and reproduction in any medium, provided the original work is properly cited. gastrojejunostomy has a better patency, but is invasive and associated with surgery-related morbidity. ${ }^{1}$ Endoscopic ultrasonography-guided gastroenterostomy (EUS-GE) using a lumen-apposing metal stent (LAMS) is a novel investigational technique, that seems to overcome the shortcomings of the current standard treatments. ${ }^{2}$ It is minimally invasive, and the risk of stent dysfunction due to migration or tumor ingrowth is considered to be low because of the features of the LAMS (bi-flanged, short in length, fully covered, and positioned away from the tumoral area). ${ }^{3}$ Here, we report two cases with early LAMS dysfunction after EUS-GE had been performed.

\section{CASE REPORTS}

\section{The first case}

A 72-year-old male (American Society of Anesthesiologists [ASA] II) presented with symptoms of vomiting and inability to eat since several weeks. Upper endoscopy revealed a large circular gastric carcinoma in the distal antrum, causing an outlet obstruction. Computed tomography (CT) revealed 


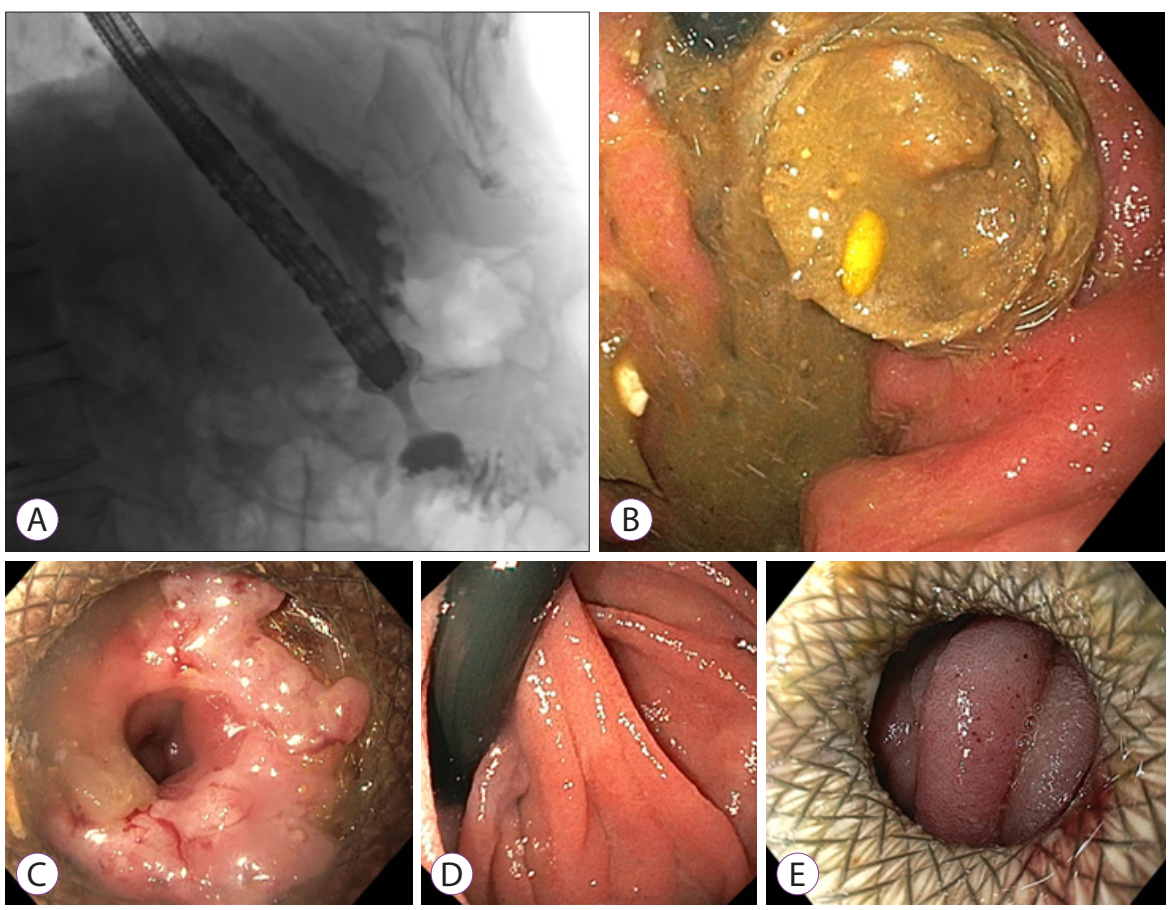

Fig. 1. The first case. (A) Lumen-apposing metal stent placement. (B) Food impaction. (C) Hyperplastic overgrowth at the distal flange. (D) Distal flange not visible from the jejunum with the nasoscope in U-turn. (E) Second lumen-apposing metal stent placement, $20 \mathrm{~mm}$ diameter.

loco-regional lymphadenopathy and multiple small bilateral liver metastasis. There were no curative options. Based on the limited extent of metastasis and a reasonable life expectancy of the patient at that time, duodenal stenting was deemed unsuitable due to the risk of re-obstruction over time. As the patient did not prefer undergoing a surgical gastroenterostomy, an EUS-GE was performed to palliate the obstructive symptoms. The direct puncture technique was performed. ${ }^{4}$ From the proximal antrum, a 15-mm LAMS (Hot AXIOS ${ }^{\prime \prime}$ Stent and Electrocautery-Enhanced Delivery System; Boston Scientific Co., Marlborough, MA, USA), the standard-diameter LAMS that was used at that moment, was successfully deployed (Fig. 1A). The patient was discharged the next day.

After three weeks, the patient returned with symptoms of early satiety. A second upper endoscopy was performed and it revealed a LAMS obstructed by food impaction (Fig. 1B). The food bolus was extracted endoscopically, and the patient was discharged. Six weeks after the LAMS placement, the patient presented again with recurrent symptoms of GOO. A third upper endoscopy revealed that the LAMS had become stenotic due to hyperplastic tissue overgrowth (Fig. 1C), and the distal flange was buried in the jejunal wall (Fig. 1D). Dilation with a 12-mm controlled radial expansion (CRE)-balloon was performed, but the patient's symptoms hardly improved.
The persisting hyperplastic tissue, covering and obstructing the distal end of the stent, was partly treated by argon plasma coagulation, but a $9.8-\mathrm{mm}$ endoscope still could not pass through the LAMS. The patient was rescheduled for a stent change. The original 15-mm LAMS was removed and replaced by a new 20-mm LAMS (Fig. 1E), ten weeks after the first LAMS placement. Palliative chemotherapy was started four days later. Because of vomiting, upper endoscopies were repeated twice, two days after and one month after initiating the chemotherapy. During both endoscopic examinations, a patent LAMS and an empty stomach were noted. Therefore, the complaints were respectively attributed to chemotherapy (which was discontinued after one cycle, following patient's wish to stop due to side effects), and to a short and transitory period of food impaction (as the patient was able to eat again spontaneously, after the last upper endoscopy). The patient suffered from a rapidly progressive disease and passed away 4.5 months after the initial LAMS placement.

\section{The second case}

A 31-year-old female (ASA I) with a history of melanoma in situ, presented with postprandial nausea and vomiting since 6 weeks. Upper endoscopy revealed external compression of the horizontal part of the duodenum. CT revealed a $55 \times 38$ - 

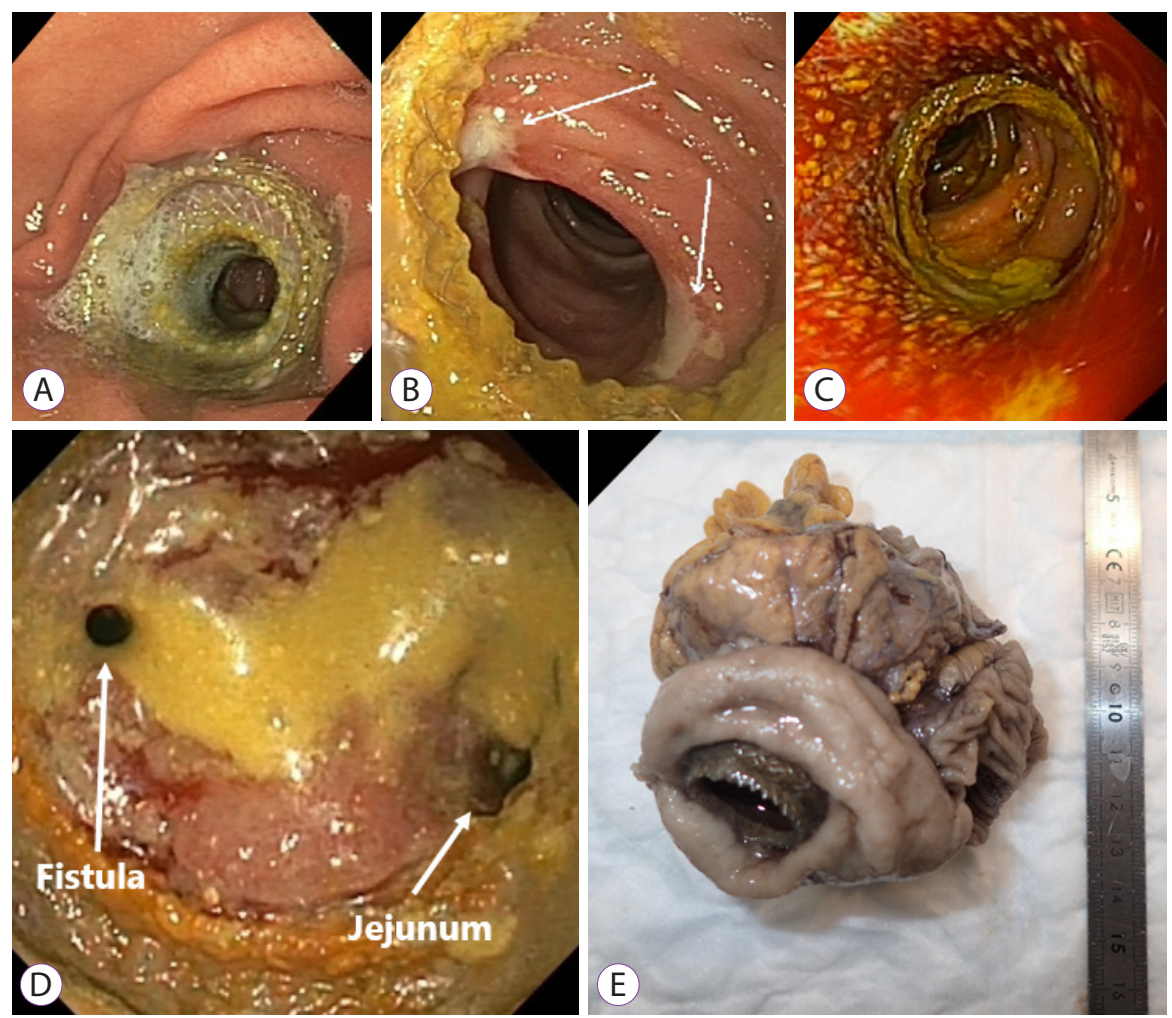

Fig. 2. The second case. (A) Relative stenosis of the lumen-apposing metal stent-before dilation. (B) Jejunal pressure ulcers (arrows). (C) Balloon dilation of relative stenosis-after dilation. (D) Gastro-colonic fistula (left arrow) and jejunum (right arrow). (E) Surgical specimen of the transverse colon and jejunum with lumenapposing metal stent in situ.

$\mathrm{mm}$ mass, originating from the uncinate process, with circular encasement of the proximal superior mesenteric artery and tumor progression in the left para-aortic region. Histological examination confirmed the diagnosis of a locally advanced pancreatic adenocarcinoma. Patient's condition was good, her life expectancy was reasonable, and induction chemotherapy with additional surgery (when tumor regression allowed this) was planned. Therefore, duodenal stenting and surgical gastrojejunostomy were not preferred. Rather, direct EUS-GE was performed, with successful placement of a 20-mm diameter LAMS (Hot AXIOS '" Stent) between the antrum and the small intestine.

Two days later, the patient complained of postprandial epigastric cramps. Upper endoscopy revealed a patent and well-positioned LAMS, which could easily be passed with the pediatric gastroscope. Analgesics were administered for reducing pain, and induction chemotherapy was initiated 16 days after LAMS placement. One day after the first chemotherapy, the patient was readmitted to the hospital because of postprandial vomiting. Upper endoscopy, three weeks after LAMS placement, revealed a patent, but suboptimally expand- ed LAMS, with the opposite side of the enteral wall blocking the distal end of the LAMS (Fig. 2A). In addition, pressure ulcers were seen at the distal flange of the LAMS (Fig. 2B). As the stent was not fully expanded to its maximal size, the subsequent lengthening of the LAMS was probably the cause of the ulcerations. CRE-balloon dilation up to $20 \mathrm{~mm}$ was performed (Fig. 2C), and the patient's symptoms improved. Two months later, however, the 5th chemotherapy cycle had to be postponed because of epigastric pain associated with worsening postprandial nausea and vomiting. A third repeat endoscopy revealed regenerative tissue overgrowth (biopsy proven) obstructing the distal end of the LAMS. Ulcerations were not visible; however, when the LAMS was passed, two small openings were identified (Fig. 2D): one to the jejunum, and a second opening suspected to be a gastro-colonic fistula (since colonic haustrae were identified after exploring the opening with the endoscope). Because the primary tumor regressed after neoadjuvant chemotherapy, a Whipple procedure (pancreaticoduodenectomy) was performed 17 weeks after the LAMS placement. During this procedure, the LAMS was identified, positioned in the stomach and attached to the transverse co- 
lon and to a jejunal loop. A right hemicolectomy, combined with a partial jejunal resection and resection of the greater curvature of the stomach, was performed to remove both the LAMS and the fistula. The surgical specimen of the resected LAMS showed the LAMS with tissues of the transverse colon and jejunum (Fig. 2E). Further pathological evaluation of the jejunum revealed the presence of gastric mucosa and ulcerations. Tissue around the stent (colon, jejunum, and distal stomach) was tumor-free. The patient experienced a complicated recovery, and was discharged after two months. Because of R1 tumor resection, adjuvant chemotherapy was indicated; however, the patients' physical condition did not allow this. Seven months after the LAMS placement (four months after surgery), the patient developed an ileus of the small intestine and was diagnosed with peritoneal carcinomatosis. She was discharged home with best supportive care.

\section{DISCUSSION}

Long-term patency of EUS-GE is crucial to maintain oral intake, avoid hospital admissions, and improve quality of life in patients with malignant GOO. Some of the currently available studies did not report any case of stent dysfunction. ${ }^{2}$ Other studies reported one or two cases of stent dysfunction, either due to food impaction, ${ }^{5}$ inflammatory tissue overgrowth, ${ }^{4}$ mesh erosion, or stent ingrowth. ${ }^{6}$ Two case reports described tissue ingrowth of a 15-mm LAMS after more than six months, due to mesh erosion or failure of stent covering. ${ }^{7.8}$ Yet, the timing of stent dysfunction was not always reported. In the present cases, patency was compromised early after the LAMS placement and with intact LAMS covering, requiring multiple repeat endoscopies.

The distal flange that was buried within the layers of the jejunal wall due to hyperplastic tissue overgrowth, as seen in the first case, occurred six weeks after the initial stent placement. A comparable case has been described recently, though it concerned the gastric flange of the LAMS and occurred after an indwelling time of 13 months. ${ }^{9}$ It resembles the so-called buried bumper syndrome, a known complication of (semi-) rigid fixation devices, that presents with hyperplastic tissue growth over the edge..$^{10}$ Likewise, in patients with pancreatic fluid collections (PFC) treated with EUS-guided drainage using LAMS, buried stent syndrome has been reported, in particular after an indwelling time of more than 4 weeks. ${ }^{11,12}$ In the second case of the present study, hyperplastic tissue overgrowth occurred after seven weeks. Here, it concerned a 20-mm LAMS. The larger diameter of the stent apparently did not protect it from obstruction.
The relative LAMS stenosis with jejunal ulceration occurred three weeks after initial LAMS placement. It could be explained by the narrowing and subsequent lengthening of the stent, causing pressure ulcerations. Ulceration at the LAMS site has been reported previously. ${ }^{13}$ Some authors therefore suggested regular evaluation of the LAMS after EUS-GE, either by CT-scan or repeated endoscopies, to reduce the incidence of adverse events such as tissue overgrowth and ulcerations. ${ }^{14}$

The gastro-colonic fistula, which occurred 11 weeks after the LAMS placement, might have resulted from suboptimal deployment and subsequent lengthening of the stent, causing prolonged pressure erosions due to friction between the transverse colon and the flanges of the gastro-jejunal LAMS. The mechanism might be similar to when LAMS is impinging adjacent vasculature causing friction and bleeding, when used for draining PFC. ${ }^{11}$ Another explanation could be that the colon was too close to the jejunum during the stent placement, and might have already been intertwined and connected to the LAMS at that stage, resulting in pressure necrosis and gradual fistulization.

In conclusion, EUS-GE stent dysfunction rates reported in literature are low. We reported two cases of early LAMS dysfunction complicating EUS-GE in patients with malignant GOO, compromising patency and causing substantial morbidity. Although EUS-GE has the potential of being a minimally invasive and durable treatment for malignant GOO, there is insufficient evidence to endorse EUS-GE as standard of care for GOO. To date, numbers and causes of LAMS dysfunction have not been clearly evaluated and elucidated, respectively. Therefore, it is important to be aware that (early) recurrent symptoms and adverse events can occur. Prospective studies with adequate follow-up and registration of adverse events are crucial to evaluate the effectiveness and safety of novel techniques such as EUS-GE.

\section{Conflicts of Interest}

Leon M. G. Moons and Frank P. Vleggaar are consultants for Boston Scientific Corporation. The authors have no potential conflicts of interest.

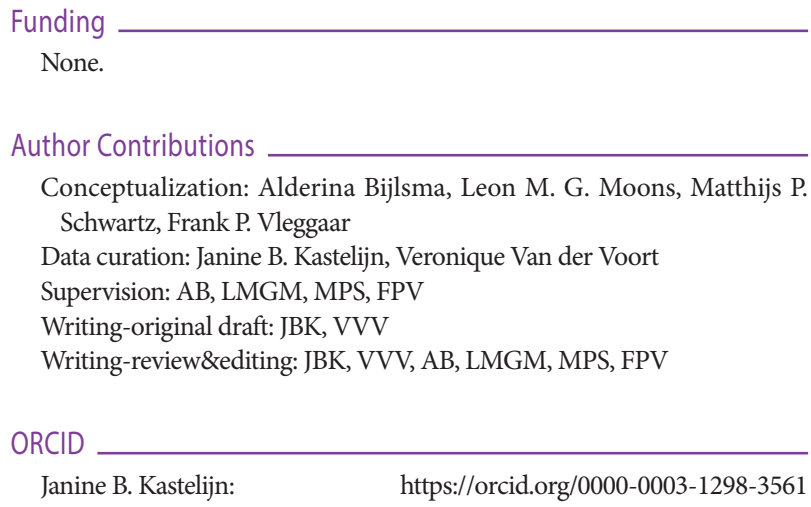


Veronique Van der Voort: Alderina Bijlsma:

Leon M. G. Moons: Matthijs P. Schwartz: Frank P. Vleggaar: https://orcid.org/0000-0003-3163-5941 https://orcid.org/0000-0002-8512-9174 https://orcid.org/0000-0002-6913-9954 https://orcid.org/0000-0003-3106-3373 https://orcid.org/0000-0001-8664-5130

\section{REFERENCES}

1. Upchurch E, Ragusa M, Cirocchi R. Stent placement versus surgical palliation for adults with malignant gastric outlet obstruction. Cochrane Database Syst Rev 2018;5:CD012506.

2. Iqbal U, Khara HS, Hu Y, et al. EUS-guided gastroenterostomy for the management of gastric outlet obstruction: a systematic review and meta-analysis. Endosc Ultrasound 2020;9:16-23.

3. Tyberg A, Perez-Miranda M, Sanchez-Ocaña R, et al. Endoscopic ultrasound-guided gastrojejunostomy with a lumen-apposing metal stent: a multicenter, international experience. Endosc Int Open 2016;4:E276-E281

4. Chen YI, Kunda R, Storm AC, et al. EUS-guided gastroenterostomy: a multicenter study comparing the direct and balloon-assisted techniques. Gastrointest Endosc 2018;87:1215-1221.

5. Kerdsirichairat T, Irani S, Yang J, et al. Durability and long-term outcomes of direct EUS-guided gastroenterostomy using lumen-apposing metal stents for gastric outlet obstruction. Endosc Int Open 2019;7:E144-E150

6. Ge PS, Young JY, Dong W, Thompson CC. EUS-guided gastroenterostomy versus enteral stent placement for palliation of malignant gastric outlet obstruction. Surg Endosc 2019;33:3404-3411.

7. Madanat L, Saumoy M, Sharaiha RZ. Endoscopic gastrojejunostomy bigger is better. Endoscopy 2018;50:E331-E332.

8. Parekh PJ, Shakhatreh MH, Yeaton P. A tale of two LAMS: a report of benign tissue ingrowth resulting in recurrent gastric outlet obstruction. Endosc Int Open 2018;6:E1390-E1394.

9. Dioscoridi L, Forti E, Pugliese F, et al. Buried lumen-apposing metal stent after gastrojejunal bypass. Endoscopy 2020;52:E134-E135.

10. Cyrany J, Rejchrt S, Kopacova M, Bures J. Buried bumper syndrome: a complication of percutaneous endoscopic gastrostomy. World J Gastroenterol 2016;22:618-627.

11. Bang JY, Hasan M, Navaneethan U, Hawes R, Varadarajulu S. Lumen-apposing metal stents (LAMS) for pancreatic fluid collection (PFC) drainage: may not be business as usual. Gut 2017;66:2054-2056.

12. Bang JY, Hawes RH, Varadarajulu S. Lumen-apposing metal stent placement for drainage of pancreatic fluid collections: predictors of adverse events. Gut 2020;69:1379-1381.

13. Irani S, Jalaj S, Ross A, Larsen M, Grimm IS, Baron TH. Use of a lumen-apposing metal stent to treat GI strictures (with videos). Gastrointest Endosc 2017;85:1285-1289.

14. Taibi A, Durand Fontanier S, Derbal S, et al. What is the ideal indwelling time for metal stents after endoscopic ultrasound-guided gastrojejunostomy? Case report of delayed iatrogenic perforation with a review of the literature. Dig Endosc 2020;32:816-822. 